\title{
Architecture of solution for panoramic image blurring in GIS project application
}

\author{
Dejan Vasić ${ }^{1}$, Marina Davidović ${ }^{1}$, Ivan Radosavljević ${ }^{2}$, and Đorđe Obradović ${ }^{2}$ \\ ${ }^{1}$ Department for Civil Engineering and Geodesy, Faculty of Technical Sciences, University of Novi Sad, Novi Sad, \\ 21000, Vojvodina, Republic of Serbia \\ ${ }^{2}$ School of Informatics and Computing, Singidunum University, Belgrade, 160622, Republic of Serbia
}

Correspondence: Marina Davidović (marina.davidovic@uns.ac.rs)

Received: 7 July 2021 - Discussion started: 20 July 2021

Revised: 7 October 2021 - Accepted: 27 October 2021 - Published: 23 November 2021

\begin{abstract}
Panoramic images captured using laser scanning technologies, which principally produce point clouds, are readily applicable in colorization of point cloud, detailed visual inspection, road defect detection, spatial entities extraction, diverse map creation, etc. This paper underlines the importance of images in modern surveying technologies and different GIS projects at the same time having regard to their anonymization in accordance with law. The General Data Protection Regulation (GDPR) is a legal framework that sets guidelines for the collection and processing of personal information from individuals who live in the European Union (EU). Namely, it is a legislative requirement that faces of persons and license plates of vehicles in the collected data are blurred. The objective of this paper is to present a novel architecture of the solution for a particular object blurring. The architecture is designed as a pipeline of object detection algorithms that progressively narrows the search space until it detects the objects to be blurred. The methodology was tested on four data sets counting 5000, 10000, 15000 and 20000 panoramic images. The percentage of accuracy, i.e., successfully detected and blurred objects of interest, was higher than $97 \%$ for each data set. Additionally, our aim was to achieve efficiency and broad use.
\end{abstract}

\section{Introduction}

The rapid advancement of the technologies used in geodesy and geomatics has opened up many possibilities in various scientific spheres. A tremendous development of information technology is one of the driving factors behind that great growth of surveying and geodesy science (Habib et al., 2020). The implementation of laser scanning technology combined with a high-precision navigation system enables 3D scanning of road infrastructure (mobile laser scanning - MLS). By using this method, time consumption is reduced (Sztubecki et al., 2020), and it is possible to obtain a significantly larger amount of information. Furthermore, the representation of a larger terrain area with a higher level of detail is enabled, as are various additional analyses and highefficiency processing of the collected data.

Reliable feature extraction from 3D point cloud data is an important phase in numerous application domains, such as traffic managing, object recognition, autonomous navigation, civil engineering and architectural projects. All those projects require reliable, quality data clearly demonstrating the real-time conditions in the field to be successfully completed. When surveying the terrain with an MLS, besides a point cloud as the main output product, the digital camera collects panoramic photographs. Each photo is associated with the appropriate position from the trajectory, and in this way the photo is matched with the point cloud (Batilovic et al., 2019). These images are gaining in importance due to the processes of visualization and different map creation. With image usage, visual inspection is facilitated and various types of damage can be detected (Davidović et al., 2021). According to Lahoti et al. (2019), maps that graphically present particular data of interest about an urban area can be generated. That has been shown to be very helpful for planners and architects in positioning future objects, regulating green areas, traffic management, forest management (Kuzmić et al., 2017), and many other things. Since images play a vital 
role in a lot of different disciplines, they have inevitably become part of collected data. These most often involve cars and pedestrians. Following GDPR (European Data Protection Regulation), those pictures are to have blurred faces of people and car license plates. In order to secure legitimate panoramic images, the detection and blurring of the abovementioned features ought to be conducted. Whilst it is clear that there are justifiable reasons for sharing multimedia data acquired in such ways (e.g., for law enforcement, forensics, bioterrorism surveillance, disaster prediction), there is also a strong need to protect the privacy of innocent individuals who are inexorably "captured" in the recordings (Ribaric et al., 2016). For instance, the average citizen in London is caught on CCTV cameras about 300 times a day (Cavalaro 2007).

Moreover, the necessity for object detection has significantly increased. The reasons for this include a growing demand for automatic vehicle identification required for traffic control, border control, access control, calculation of parking time and payment, and searching for stolen cars or unpaid fees, along with the requirement for reliable identification considering a complex diversity of circumstances, e.g., different lighting conditions, the presence of random or structured noise in the plate, its size and type of characters, and nationality-specific features (Kasaei et al., 2010).

This study focuses on automatic object detection from panoramic images, obtained by mobile mapping technology, which is followed by the blurring of those objects.

The remainder of the paper is divided into four sections. The section "Related work" provides a descriptive summary of certain methods that have been implemented and tested in the area of automatic object detection and blurring. The section "Materials and methods" offers an insight into the proposed methodology. Experimental results are discussed in the section "Results and discussion". Conclusions and further work are presented in the last section.

\section{Related work}

Some studies related to the paper topic are presented in this section. Several authors define object detection and its significance (Demir, 2014; Božić-Štulić et al., 2018; Radović et al., 2017). Some authors deal with object detection in general, while others point out the detection of particular spatial entities. In recent years, deep learning approaches using features extracted by convolutional neural networks (CNNs) have significantly improved the detection accuracy. Sommer et al. (2017) propose a deep neural network derived from the Faster R-CNN approach for multi-category object detection in aerial images. The detection accuracy was shown to be capable of improvement by replacing the network architecture with the one specially designed for handling small objects. The Faster R-CNN approach for medium-sized objects was elaborated in Zhang et al. (2016). Božić-Štulić et al. (2018) used a pre-trained Faster R-CNN model for the detection of minor deformations from images obtained by UAV (unmanned aerial vehicle) surveying technology. Radović et al. (2017) details the procedure and parameters used for the training of CNNs on a set of aerial images for object recognition. The results show that by selecting a proper set of parameters, CNN can detect and classify objects with a high level of accuracy $(97.5 \%)$ and computational efficiency. In He et al. (2018), Mask R-CNN authors present an extension of Faster R-CNN called Mask R-CNN that can estimate both bounding boxes and segmentation masks in a single pass without major computation overhead. The extension proved to be simple to train and able to generalize well; thus it can be used for tasks other than bounding box and segmentation estimation.

According to Deb and Jo (2009), the vehicle license plate detection from vehicle images is a challenging task due to multi-style plate formats, viewpoint changes and the nonuniform outdoor illumination conditions during image acquisition. A real-time multiple license plate detection algorithm is described in Asif et al. (2016). The authors used color components to identify license plate regions. Experimental results show that the proposed method accurately detects $93.86 \%$ of these elements. The edge-feature-based method uses edge detection and morphological operations to find a rectangular candidate plate and then aspect ratio to filter the candidate regions. While this approach can work in many cases, the authors of Chuang et al. (2014) showed that skewed plates and small plates cannot be detected. Hamid and Shayegh (2013) advises the use of edge detection and morphological operations to identify potential license regions followed by connected component operation to identify the license plate location. Although the correct recognition rate was reported to be $98.66 \%$, this method requires multiple steps. Namely, the obtained image needs to be converted into a binary mode first and only then is the algorithm conducted. Also, the acquired time from input until final output is not mentioned. Wang and Lee (2003) detected probable license plate regions from the gradients of the input car images. Then, this element was separated into several adjacent regions, and the one with the largest possible value was chosen. Experimental results show that the rotation-free character recognition method can achieve an accuracy rate of $98.6 \%$. The flow of the suggested algorithm was the manual detection of character features that are nonsensitive to rotation variations. A region-based license plate detection method was described in Jia et al. (2007), where a mean shift procedure is applied in a spatial-range domain to segment a color vehicle image in order to get candidate regions. License plates adhere to a unique feature combination of rectangularity, aspect ratio and edge density. These three features are defined and extracted in order to decide if a candidate region contains this object. The drawback of this method is the difficulty of detecting license plates in cases 
where vehicles and their respective plates are of a similar or the same color.

Human body detection presents a number of challenges such as extracting meaningful features to capture a wide range of poses and human appearances (Deb and Jo, 2009). Most current work on human detection in color images encompasses a variety of feature descriptors and classifiers. Most notable people detection methods are based on the histogram of oriented gradient (HOG) feature descriptors (Dalal and Triggs, 2005). Dense descriptors comprising blocks with multiple histograms of image gradients are classified as human/non-human using a linear support vector machine (SVM). The histogram of image gradients is constructed and scaled for each cell. The final search window descriptor is a vector of concatenated block histograms. Although this method has been proven as reasonably efficient, there is still room for optimization and further speed-up in detections. Miezianko and Pokrajac (2008) documented a method for detecting people in low-resolution infrared videos. The suggested method is based on extracting gradient histograms from recursively generated patches and, subsequently, computing histogram ratios between the patches. Each set of patches was defined in terms of relative position within the search window, and each set was then recursively applied to extract smaller patches. The major objective of this study was to incorporate motion detection and tracking into the existing system and to limit the search in the future. Breckon et al. (2012) gave an account of a combined autonomous system for surveillance and human detection, which can also be applied to vehicle detection, using optical and thermal images. This approach primarily detects the initial segments within the scene that might contain an object. Afterwards, isolated segments are extracted, supplying a basis for secondary object classification to be carried out. As this method does not take place in real-time, Gilmore et al. (2011) suggested an almost real-time detection algorithm, founded on digital, infrared thermal imagery. The objective was to achieve pedestrian candidate selection and detection. The focus of Vu et al. (2006) is an event recognition system employing face detection and tracking combined with audio analysis. Three-dimensional contexts, such as zones of interest and static objects, were recorded in a knowledge base, and 3D positions were calculated for mobile objects using calibration matrices. The major flaw of this mechanism is the fact that substantial changes in lighting conditions occasionally prevent the system from detecting people correctly.

Nowadays, there is a growing trend in image blurring, so various authors have paid considerable attention to researching this area. Farid et al. (2018) described content-adaptive blurring $(\mathrm{CAB})$. In $\mathrm{CAB}$, a multi-focus image is iteratively blurred in such a manner that only the focused regions get blurred, whereas the defocused regions receive little or no blur at all. If blurring degrades the quality of a local image region more than by an allowable limit, that region is not blurred and exempted from further blurring. Thus, the de- focused regions are preserved while the focused regions get blurred. In Chiang et al. (2016) different image types were exploited for multiple object recognition by means of focusing and blurring. The focusing and blurring step applies image processing techniques to focus on the most important objects and blur out the rest of the image with either vignette, blur or bokeh, using the identified object bounding boxes. A method named inhomogeneous principal component blur (IPCB) was proposed in Du et al. (2011). It adaptively blurs different pixels of a license plate by taking into account the prior distribution of sensitive information. The blurring is based on the principal component analysis (PCA) approach - the original plate's area is substituted by a reconstructed area that is obtained by applying a smaller number of eigenvectors. The detection of faces and license plates in Google Street View footage was demonstrated in From et al. (2009), where de-identifications were simply done by blurring the detected locations. A simplified version of the face detector based on a fast sliding-window approach over a range of window sizes was used for the detection of license plates. They belong to a large family of sliding-window detectors, such as Schneiderman and Kanade (2001) and ViolaJones detectors (Viola and Jones, 2004). The authors have reported that a completely automatic system detected and sufficiently blurred $94 \%-96 \%$ of the total number of license plates and more than $86 \%$ of faces in evaluation sets sampled from Google Street View imagery.

\section{Materials and methods}

The solution proposed in this paper is composed of four software components:

- a project management module;

- a vehicles and people detection module;

- a license plate detection module;

- a blurring module.

The project management module is a web application that provides a user interface (UI) necessary to coordinate the blurring process. Through this interface, it is possible to setup and monitor the blurring process and manually correct the obtained results. Besides the UI, the project management module is responsible for distribution of configurations and tasks to the rest of the modules. It is also the main collection point for the results produced by the aforementioned modules and the final processing unit which encompasses the blurring module. The back end of the project management module was implemented using the Flaks web application framework (The Pallets Projects, https://palletsprojects.com/p/flask, last access: 1 February 2021), ZeroMQ and the OpenCV library (ZeroMQ, https://zeromq.org, last access: 2 February 2021; Open Source Computer Vision Library, https://opencv.org, 
last access: 1 February 2021). The back end exposes two ZeroMQ TCP client sockets which are utilized to send processing requests and to receive responses from other modules. The first socket is used to communicate with the vehicles and people detection module, whilst the second one is intended for the communication with the license plate detection module. The front end of the project management module was implemented using the Angular framework and OpenLayers library (Angular, https://angular.io, last access: 5 February 2021; OpenLayers, https://openlayers.org, last access: 5 July 2021).

The vehicle and people detection module and the license plate detection module are responsible for the extraction of the bounding boxes from the raw images supplied by the project management module. These modules can contain one or more processing nodes. Each processing node exposes a TCP server ZeroMQ socket. The server sockets accept requests from the project management module. Each request contains the actual image that has to be processed. After its processing, the same socket is used to reply to the project management module with a message containing the bounding boxes extracted from the input image.

The processing nodes of the vehicle and people detection module have been implemented as a neural network trained for object detection. This network was implemented based on the TensorFlow Object Detection API (Git Hub). The model used from the API was the Faster R-CNN ResNet101 trained on the COCO data sets. This model was trained to detect all of the 80 classes in the COCO data set, which include vehicles and people (Lin et al., 2015). The output of this neural network consists of the bounding boxes and classes of the detected objects. Although the input into the processing node is the whole image, the input into the network comprises overlapping patches of the original image scaled down to the resolution of 1000 pixels by 600 pixels. The minimum overlap of the patches is $50 \%$ which ensures that no objects are skipped or partially detected. The main advantage of splitting the image into smaller patches is the reduction of the required computational resources. The downside of this approach is the creation of multiple overlapping bounding boxes for the same detected object. Once returned to the project management module, these overlapping boxes have to be merged into a single bounding box. The process of merging was done using the non-maximum suppression algorithm (Neubeck and Van Gool, 2006). The processing nodes of the license plate detection module were implemented by the means of the same model as those of the vehicle and people one. However, the neural network used in the license plate detection nodes was additionally trained to detect license plates in the images of vehicles. The training was conducted on a data set containing 1000 examples of vehicle images which contained a license plate. Each license plate was supplied with both the image and its bounding box. The bounding boxes and paths of the images they belonged to were stored in Pascal VOC XML format. The number of training examples was chosen empirically and proven sufficient for the neural network to learn distinctive patterns of license plates. The input into the license plate detection module consists of the images of vehicles provided by the project management module. These were generated by the project management module from raw images by extracting patches corresponding to the bounding boxes of vehicles detected in the vehicle and people detection module. The output of this module comprises the bounding boxes of license plates detected on the input patches with their coordinates adjusted with respect to the original image.

The blurring module is an integral part of the project management module and is responsible for blurring the areas designated by both the vehicle and people detection module and the license plate detection module. The input into this module represents raw images and the bounding boxes obtained by processing images in the aforementioned modules. A set of bounding boxes, detected in the same image, was used for each corresponding input image to determine the area of the image that has to be blurred. If the bounding box corresponds to a license plate, the area of the image covered by the bounding box is immediately blurred using the Gaussian blur. However, if the bounding box corresponds to a person, the blurring module first detects the face of that person and then blurs the area of the detected face using the Gaussian blur. The idea was to avoid unnecessary blurring of faces on billboards and similar public displays - the ones that are recognized as faces but they do not belong to the group prone to the risk of identity misuse or are not considered sensitive personal information. This kind of procedure aims to facilitate the processing performed by the algorithm since it does not require a search of all images but just the detected areas. This makes the methodology described in this paper faster and more precise. The face detection was done using the Haar classifier provided by the OpenCV library. The classifier was trained to detect faces from the frontal view. The output of the blurring module presents blurred images and bounding boxes of the blurred areas. These two elements are stored in the output folder defined in the project management module.

An overview of the proposed innovative architecture of the solution as well as the data flow between components is shown in Fig. 1.

A major goal of implementing the presented architecture was to achieve a considerable boost in performance of the license plate and face detection algorithms by creating a pipeline that would first localize major regions of interest, such as vehicles and pedestrians and then pass those regions to the more specialized components of the pipeline that would be tasked with detecting license plates and faces on those smaller regions of interest, effectively simplifying the problem of license plate and face detection.

The main advantage of the architecture of the solution elaborated in this paper is the ease with which it can be scaled up in order to reduce the computation time necessary to process large amounts of images. This scaling can be done by 


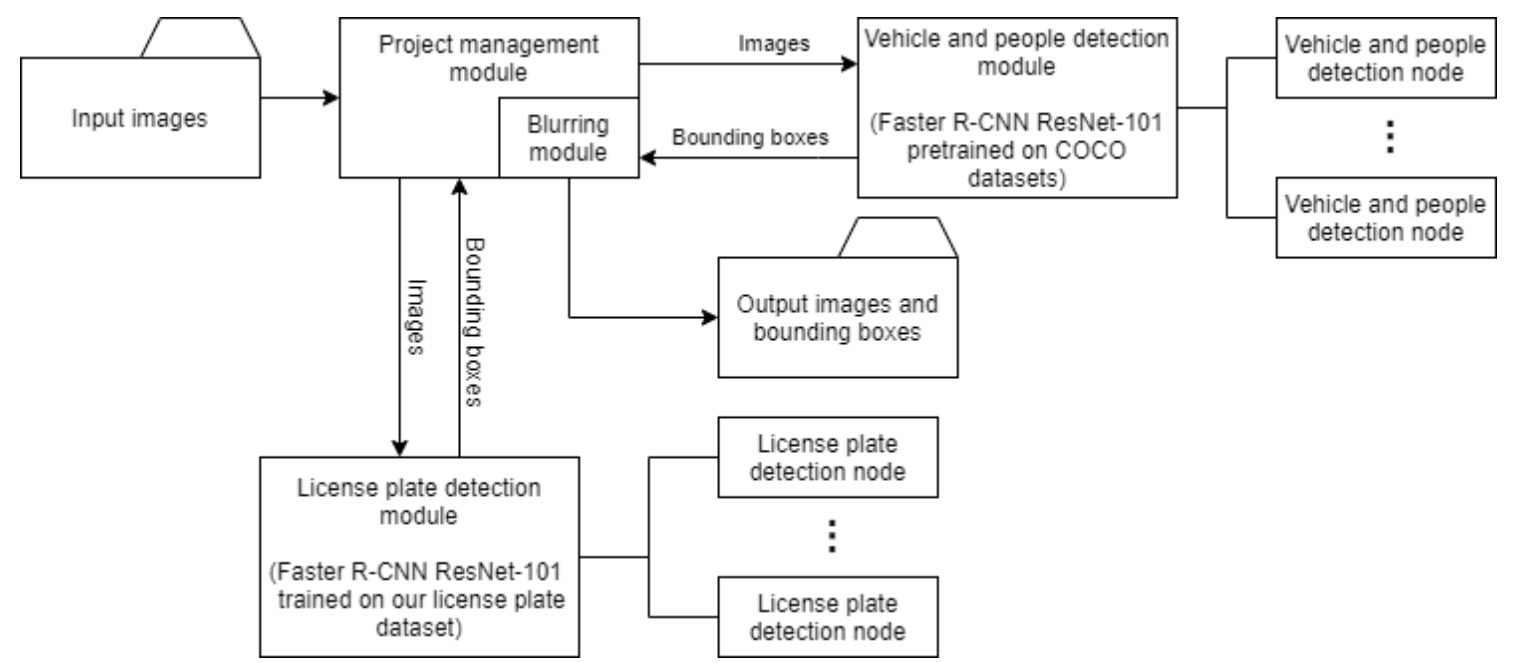

Figure 1. Architecture of the solution.

Table 1. Specifications of computer used for experiment.

\begin{tabular}{ll}
\hline Component & Type \\
\hline CPU & Second-generation Ryzen ${ }^{\mathrm{TM}}$ 7 2700X desktop processor $-16 \mathrm{~T} @ 3.7 \mathrm{GHz}$ \\
RAM memory & $2 \times$ Kingston HyperX Predator CL16, HX432C16PB3/16 - 2 $\times 16 \mathrm{~GB} @ 3200 \mathrm{MHz}$ \\
Graphic card & $2 \times$ Nvidia GeForce GTX 1660 SUPER -6 GB \\
SSD & Crucial MX500 1 TB 3D NAND SATA M.2 CT1000MX500SSD4 - 1 TB \\
HDD & SEAGATE 8 TB-SATA III-128 MB-HDD - ST8000AS0002 - 8 TB \\
\hline
\end{tabular}

deploying additional processing nodes. Each node can be deployed on a dedicated computer or it can share a single computer with other nodes. If the nodes are used on dedicated computers, it is necessary to connect those computers in a computer network to allow them to share data. Due to the amount of data that is shared between the nodes during the processing, it is necessary to provide a network infrastructure with a bandwidth of no less than $1 \mathrm{Gbits}^{-1}$ to achieve an optimal performance of the solution. Also, the nodes were implemented to utilize CUDA-capable GPUs to increase the speed of both training and exploitation of the model. Thus, each node requires a dedicated CUDA-capable GPU in order to be successfully used.

\section{Results and discussion}

Nowadays, as projects are requesting more precision and details, the input data are requested to have more details too. Colorization of the point cloud is performed with the help of collected images, and this has helped a more efficient extraction and element recognition. Additionally, all road defects like cracking, potholes, patching, surface chip loss, and others can easily be managed with image usage. Often, the results of mobile mapping projects are published on public servers. Matching the photographs to point clouds and pub- lishing them on a web platform offers a significant advantage. This convenience is reflected in the fact that while looking at the point cloud, photographs can be observed at the same time allowing any possible doubts about the particular terrain situation to be resolved (Batilović et al., 2019). In order to use images for the above-discussed purposes, they have to be GDPR-compliant; i.e., blurring of faces and license plates is required to ensure privacy protection. Consequently, the suggested mechanism for object detection and blurring has a substantial impact on diverse projects.

This section focuses on testing the methodology explained in the previous section. In order to verify the validity and accuracy of the suggested solution architecture, several experiments were carried out. Four data sets containing 5000, 10000, 15000 and 20000 images were used in each trial. They represent a collection of panoramic images with the resolution of 8000 pixels $\times 4000$ pixels, obtained by mobile mapping scanning with the Trimble MX9 system. The fact that people's faces and license plates are taken at a different angle and have divergent size and position in panoramic images has made this experiment more challenging. Additionally, the project does not encompass uniform - but different vehicle types: cars, trucks, vans, motorcycles, etc. The computer performances used for conducting these tests are presented in Table 1, while their results are given in Table 2. 
Table 2. Experimental results.

\begin{tabular}{llllll}
\hline Data set & $\begin{array}{l}\text { Automatic blurring } \\
\text { process duration } \\
{[\mathrm{s}]}\end{array}$ & $\begin{array}{l}\text { Quality control (QC) } \\
\text { duration } \\
{[\mathrm{min}]}\end{array}$ & $\begin{array}{l}\text { Number of detected } \\
\text { objects in automated } \\
\text { process } \\
\text { [pcs] }\end{array}$ & $\begin{array}{l}\text { Number of detected } \\
\text { objects in QC process } \\
{[\mathrm{pcs}]}\end{array}$ & $\begin{array}{l}\text { Percentage of success- } \\
\text { fully detected objects } \\
{[\%]}\end{array}$ \\
\hline 5000 & 4860 & 105 & 13478 & 117 & 929 \\
10000 & 9900 & 170 & 26152 & 546 & 97.14 \\
15000 & 18240 & 270 & 39688 & 845 & 98.64 \\
20000 & 19380 & 444 & 52855 & 98.43 \\
\hline
\end{tabular}

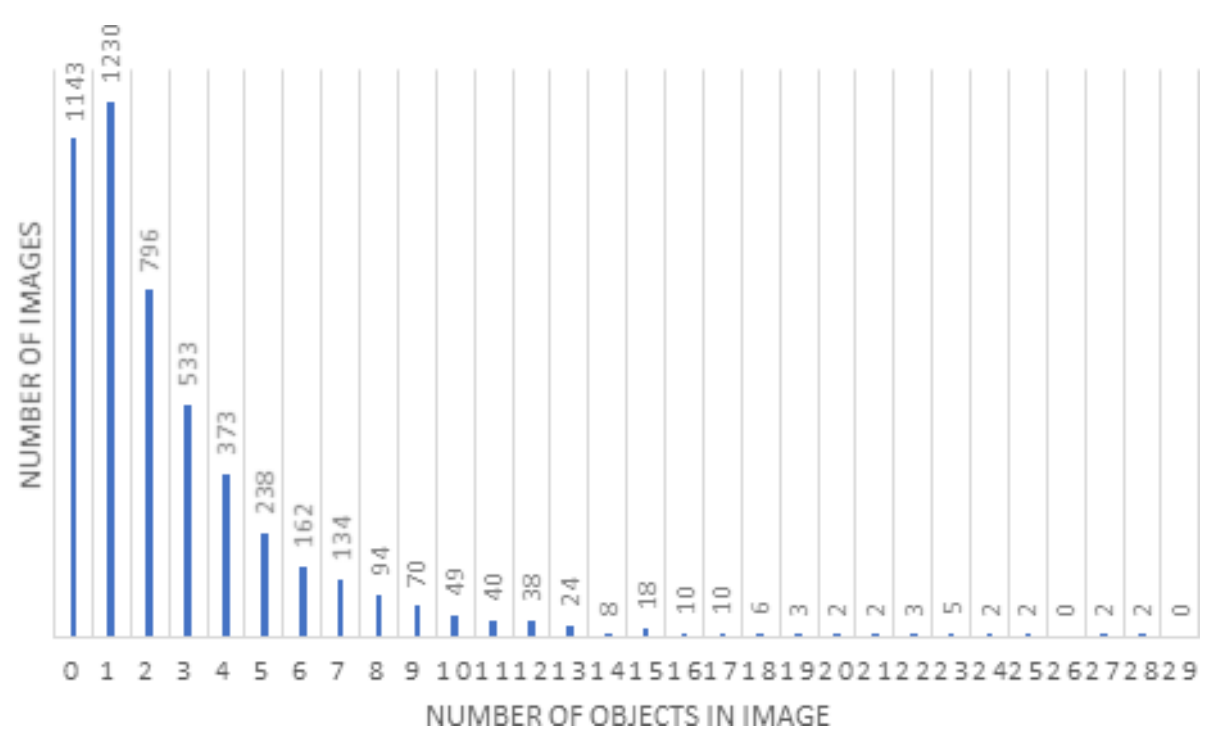

Figure 2. Average distribution of objects in images - data set with 5000 images.

The conducted experiment showed that this methodology is highly precise and is able to perform detection and blurring more efficiently than most of the solutions presented in Saidallah et al. (2021). Additionally, it has outperformed some commercially developed software with respect to its accuracy and detection and blurring speed. The software used in this study was tested four times - for four independent panoramic image data sets.

In the first data set, counting 5000 panoramic images, there were 13478 automatically detected objects in images. During the manual blurring process, i.e., quality control, $117 \mathrm{ob}-$ jects were additionally detected and blurred. Taking this into account, the percentage of successfully blurred objects was $99.14 \%$. This figure was obtained by dividing the number of automatically detected objects with the total number of objects (automatically plus manually detected). In this example, it means $13478 /(13478+117) \times 100 \%=99.14 \%$.

Figure 2 presents the ratio of the number of objects in the images and the total number of images. Here, it can be seen that some images had no objects at all. In this data set, this number was 1143. These include parts of rural areas, e.g., forest roads where there were no cars or pedestrians. On the other hand, there are a lot of images counting multiple objects. The maximum number of objects in one image was 28 .

The second data set with 10000 panoramic images contains 26152 objects detected by the proposed algorithm and 729 more objects manually found during quality control. The success percentage equals $97.29 \%$. Figure 3 illustrates a distribution chart of detected objects in this data set. It can be seen that the maximum number of detected objects in one image was 29, while the number of images where there were no bounding boxes was 2371 .

The third data set consists of 15000 panoramic images. There were 39688 objects detected and blurred, while 546 objects were found manually. The percentage of successfully blurred images was $98.64 \%$. Figure 4 represents a distribution chart of detected objects in images of this data set. The highest number of objects detected in one image equals 29, while 3479 images showed no detected objects at all.

The last data set tested consists of 20000 panoramic images. The proposed approach produced 52855 objects which were detected and blurred, while 845 objects were found manually. Figure 5 shows a distribution chart of detected objects in the fourth image data set, where the maximum num- 


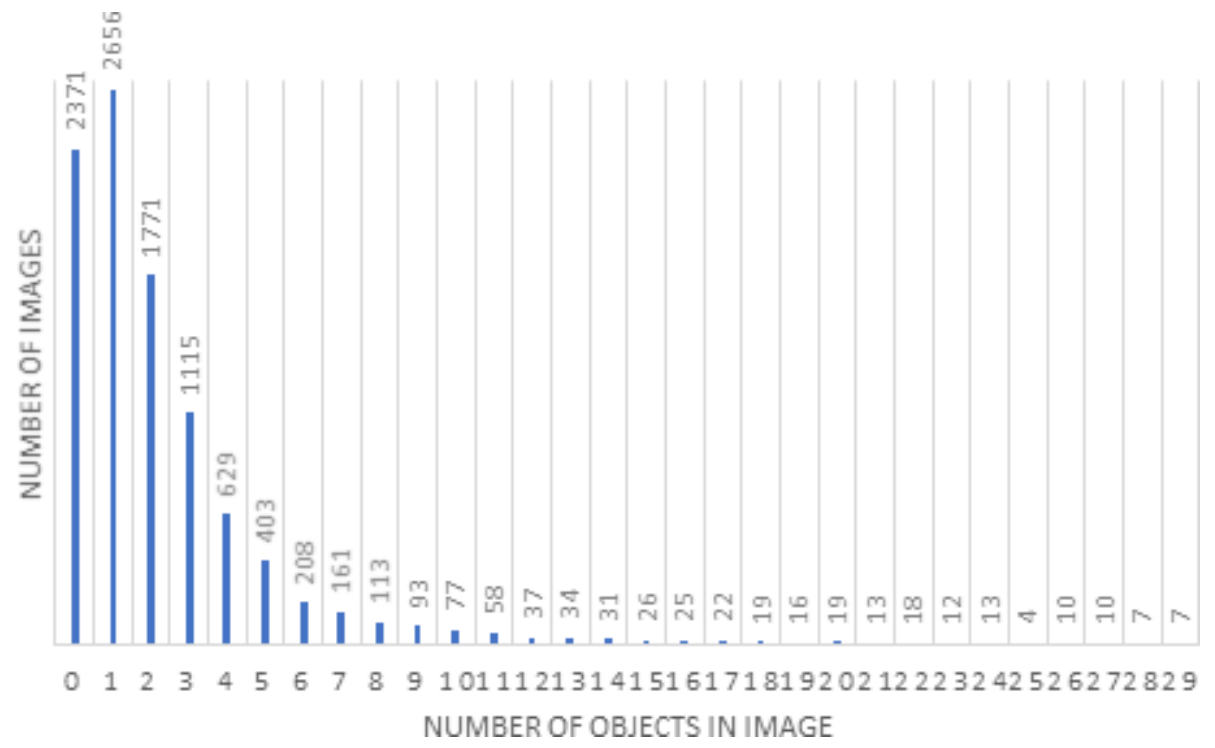

Figure 3. Average distribution of objects in images - data set with 10000 images.

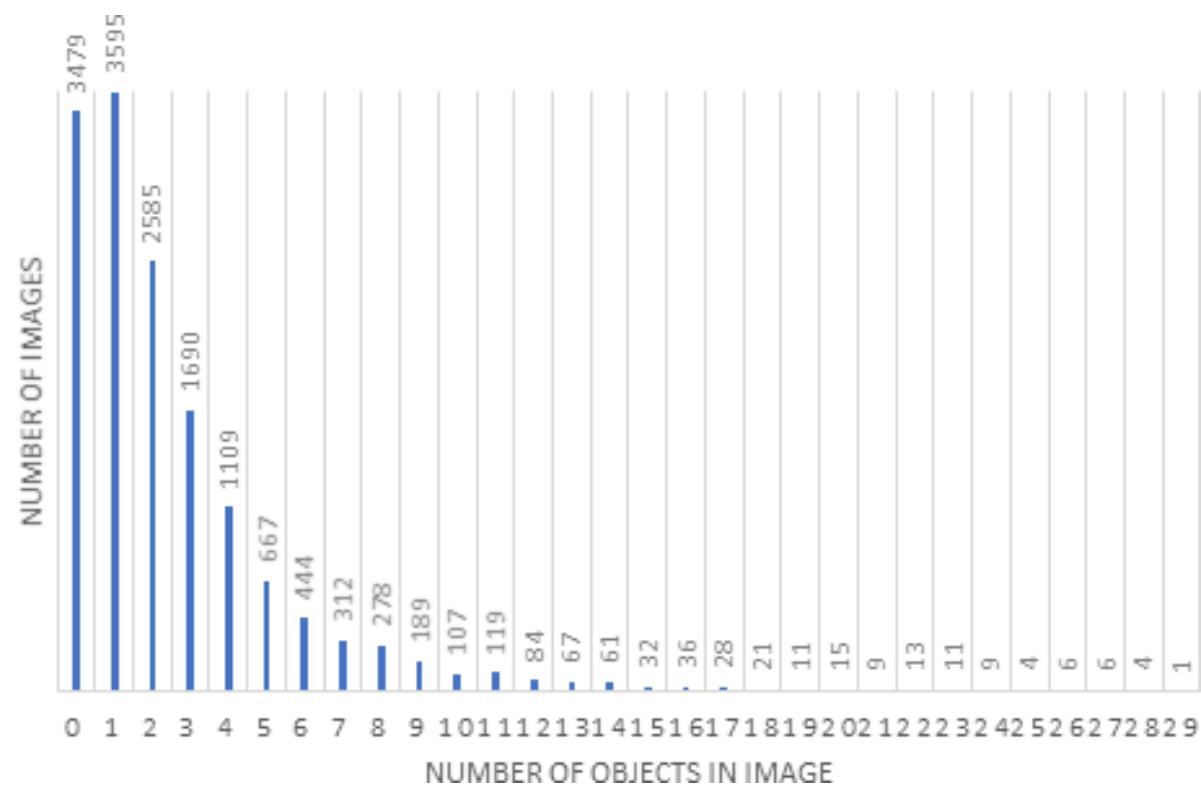

Figure 4. Average distribution of objects in images - data set with 15000 images.

ber of detected objects in one image was 29 , while $4320 \mathrm{im}$ ages had no objects detected.

Some examples of positive detected faces and license plates are presented in Fig. 6, while some of the false detected or non-detected objects can be seen in Fig. 7.

The main limitation of the suggested architecture of the solution for image processing was identified when detecting white cars because the majority of license plates are white as well. The same color makes it more difficult for the algorithm to execute detection successfully.

\section{Conclusions}

The increasing need for images in mobile mapping projects is highlighted in this paper. Since images are very often used, blurring of faces and license plates is required to comply with data protection laws. According to Ribaric et al. (2016), privacy is one of the most important social and political issues in contemporary information society, characterized by a growing range of enabling and supporting technologies and services. Amongst these are communications, multimedia, biometrics, big data, rapid development of cloud storage (Wang et al., 2021), data mining, internet, social networks 


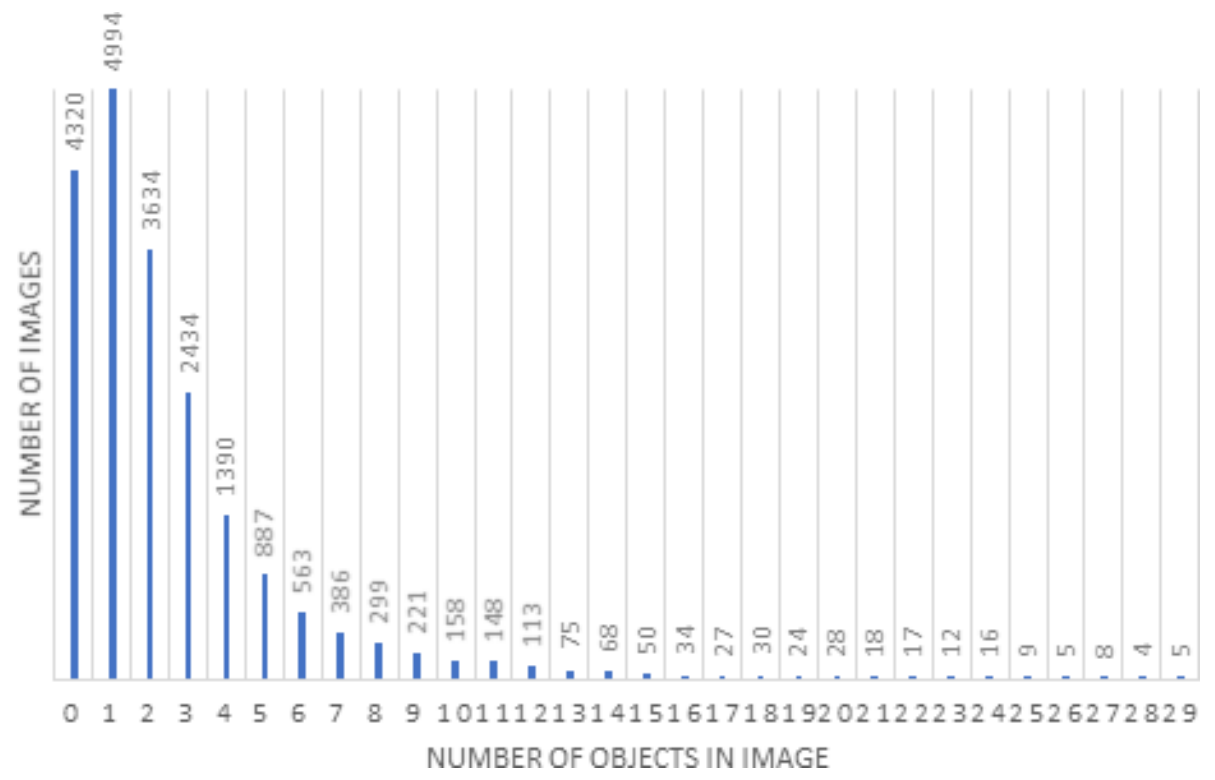

Figure 5. Average distribution of objects in images - data set with 20000 images.

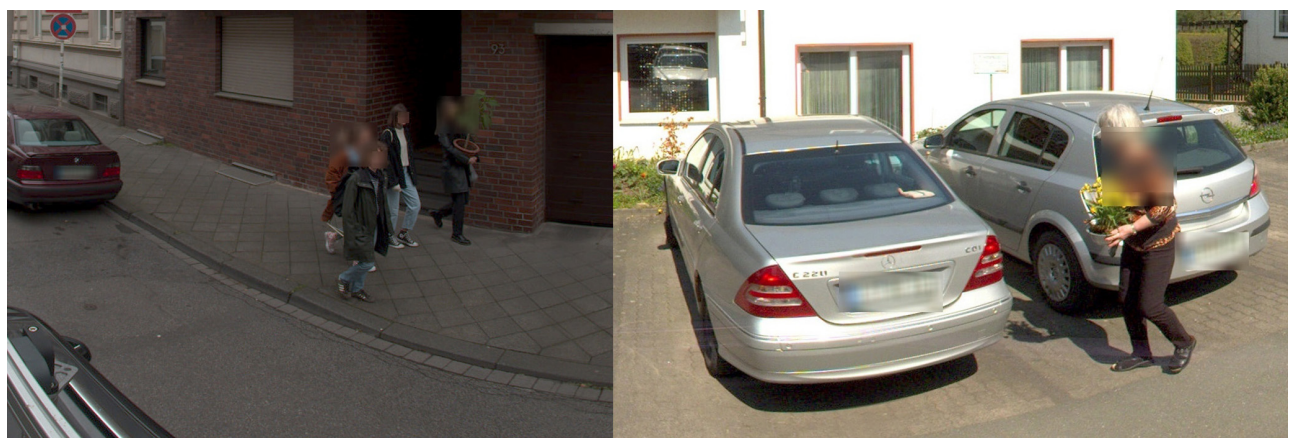

Figure 6. Examples of positive blurred panoramic images.

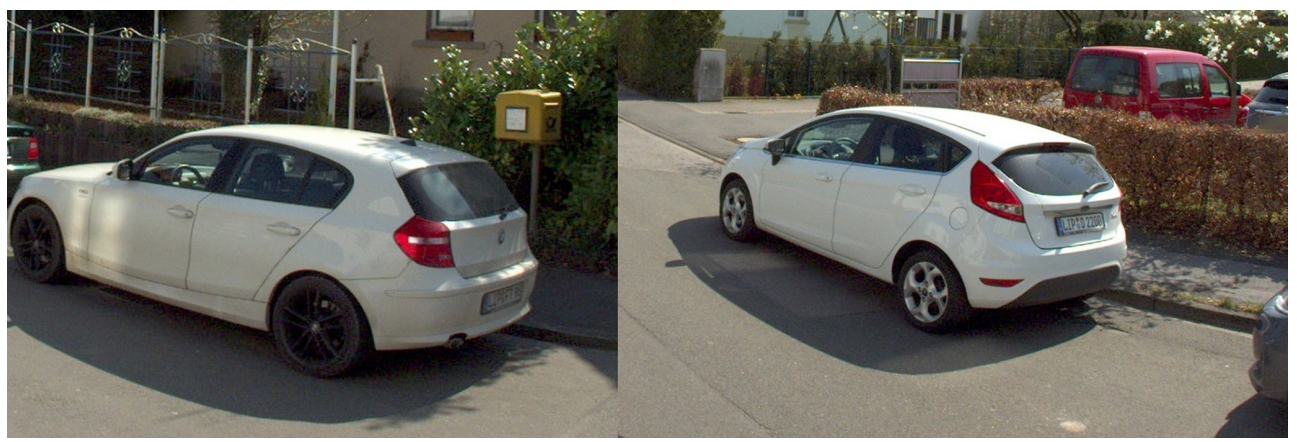

Figure 7. Examples of negative blurred panoramic images.

and audio-video surveillance. Each of these can potentially provide the means for privacy intrusion. Therefore, this article suggests a reliable method for the detection and blurring of these particular objects.

The experiment evaluating the applicability of the proposed software was conducted on four different data sets containing urban and rural area panoramic images. The total number of tested panoramic images was 50000 . These images were obtained from laser scanning of roads with a Trimble MX9 device. The success rate results for each data set were as follows: the data set with 5000 panoramic images ensured an accuracy of $99.14 \%$, the one with 10000 
panoramic images had a $97.21 \%$ accuracy, the one comprising 15000 panoramic images achieved $98.62 \%$, and the data set with 20000 images generated a $98.40 \%$ success rate.

Several major advantages of this approach were identified. The use of the described architecture drastically reduced the false positive detection of license plates and faces by utilizing a two-step approach which first detects the vehicles and pedestrians, and then proceeds to search for license plates and faces only in the areas labeled as vehicles or pedestrians. This not only reduced the false positive detections but also resulted in a high percentage of positively detected and blurred elements. Additionally, the presented algorithm was proven remarkably effective in cases when the aforementioned objects of interests had different angles, positions, colors and sizes. Moreover, the usage is very simple since it just requires the user to put the images that should be blurred into a defined folder and start the blurring machine. Next, its potential is great because it is able to use images of different size, resolution, extension, etc. Finally, its processing speed is extremely convenient as it takes less than $1 \mathrm{~s}$ to detect and blur all faces and license plates from one panoramic image.

Future work will involve three focal points. To begin with, the software will be improved in terms of detecting license plates of the same color as the vehicle they are attached to. Also, since bounding boxes are upright rectangles covering a slightly larger area than the object of interest, working on more precise bounding boxes will be the next stage of further research. They will be marking only the license plate area, taking into account its angle and tilt. In that way, unnecessary parts of the image will not be blurred, and the quality of the output image in general will be better. Eventually, the plan is to upgrade this methodology with traffic sign detection. Automatic detection of traffic signs with assigned attributes such as height, sign dimension or type of sign would make a noteworthy contribution to the quality and speed in the feature extraction domain.

Data availability. The data presented in this study are available on an FTP server that can be accessed per FileZilla (Vasić et al., 2021). The log-in parameters are as follows: IP - 188.2.72.204; user Dataset; pass - Dataset@DD2021; port-21. The data may be used only in accordance with GDPR.

Author contributions. DV and MD were responsible for conceptualization. IR developed the methodology and carried out review and editing of the paper. ĐO maintained software, was responsible for data curation and supervised the work. IR and MD were responsible for validation. DV carried out formal analysis and was responsible for resources and visualization. MD was responsible for investigation and prepared the original draft. All authors have read and agreed to the published version of the paper.
Competing interests. The contact author has declared that neither they nor their co-authors have any competing interests.

Disclaimer. Publisher's note: Copernicus Publications remains neutral with regard to jurisdictional claims in published maps and institutional affiliations.

Acknowledgements. The original data for algorithm testing were provided by the DataDEV company, Novi Sad, Republic of Serbia. The paper presents part of the research realized within the project "Multidisciplinary theoretical and experimental research in education and science in the fields of civil engineering, risk management and fire safety and geodesy" conducted by the Department of Civil Engineering and Geodesy, Faculty of Technical Sciences, University of Novi Sad.

Review statement. This paper was edited by Fernando Nardi and reviewed by two anonymous referees.

\section{References}

Asif, M., Chun, Q., Hussain, S., and Fareed, M.: Multiple Licence Plate Detection for Chinese Vehicles in Dense Traffic Scenarios, IET Intell. Transp. Sy., 10, 535-544, 2016.

Batilović, M., Vasić, D., Kuzmić, T., and Davidović, M.: Importance of Image and Point Cloud Matching of Road Infrastructure Feature Extraction, in: Proceedings of FIG Working Week 2019 "Geospatial information for a smarter life and environmental resilience", Hanoi, Vietnam, 2019.

Božić-Štulić, D., Kružić, S., Gotovac, S., and Papić, V.: Complete Model for Automatic Object Detection and Localisation on Aerial Images using Convolutional Neural Networks, Journal of Communications Software and Systems, 14, 82-90, 2018.

Breckon, T., Han, J., and Richardson J.: Consistency in MultiModal Automated Target Detection Using Temporally Filtered Reporting, in: Proceedings of the International Conference SPIE Electro-Optical Remote Sensing, Photonic Technologies and Applications VI, Edinburgh, UK, 2012.

Cavallaro, A.: Privacy in Video Surveillance, IEEE Signal Proc. Mag., 24, 168-169, 2007.

Chiang, H., Ge, Y., and Wu, C.: Multiple Object Recognition with Focusing and Blurring, Lectures from the Course Convolutional Neural Networks for Visual Recognition, Stanford University, Stanford, USA, 2015-2016.

Chuang, C.-H., Tsai, L.-W., Deng, M.-S., Hsieh, J.-W., and Fan, K.C.: Vehicle Licence Plate Recognition Using Super-Resolution Technique, in: Proceedings of 11th IEEE International Conference on Advanced Video and Signal Based Surveillance, Seoul, South Korea, 2014.

Dalal, N. and Triggs, B.: Histograms of Oriented Gradients for Human Detection, in: Proceedings of the International Conference IEEE Computer Society Conference on Computer Vision and Pattern Recognition, Washington, USA, 2005.

Davidović, M., Kuzmić, T., Vasić, D., Wich, V., Brunn, A., and Bulatović, V.: Methodology for Road Defect Detection and Admin- 
istration Based on Mobile Mapping Data, CMES-Comp. Model. Eng., 129, 207-226, 2021.

Deb, K. and Jo, K.-H.: A Vehicle License Plate Detection Method for Intelligent Transportation System Applications, Cybernet. Syst., 40, 689-705, 2009.

Demir, N.: Various Building Detection Methods with the Use of Image and Lidar Data, Tehnički vjesnik, 21, 341-349, 2014.

Du, L. and Ling, H.: Preservative License Plate De-identification for Privacy Protection, in: Proceedings of the International Conference on Document Analysis and Recognition, Beijing, China, 2011.

Farid, M., Arif, M., and Somaya, A.: Multi-Focus Image Fusion Using Content Adaptive Blurring, Inform. Fusion, 45, 96-112, 2018.

From, A., Cheung, G., Abdulkader, A., Zennaro, M., Wu, B., Bissacco, A., Neven, H., and Vincent, L.: Large-scale Privacy Protection in Google Street View, in: Proceedings of the IEEE 12th Int. Conf. on Computer Vision, Kyoto, Japan, 2009.

Gilmore, E., Ugbome, C., and Kim, C.: An IR-based Pedestrian Detection System Implemented with Matlab-Equipped Laptop and Low-Cost Microcontroller, International Journal of Computer Science \& Information Technology, 5, 79-87, 2011.

Habib, M., Alzubi, Y., Malkawi, A., and Awwad, M.: Impact of Interpolation Techniques on the Accuracy of Large-Scale DEM, Open Geosci., 12, 190-202, 2020.

Hamid, R. A. and Shayegh, R.: New Method for Optimization of License Plate Recognition system with Use of Edge Detection and Connected Component, in: Proceedings of 5th ICCKE International Conference on Computer and Knowledge Engineering, Mashad, Iran, 2013.

He, K., Gkioxari, G., Dollár, P., and Girshick, R.: Mask R-CNN, Computer Vision and Pattern Recognition, 2018.

Jia, W., Zhang, H., and He, X.: Region-based license plate detection, J. Netw. Comput Appl., 30, 1324-1333, 2007.

Kasaei, H., Kasaei, M., and Kasaei, S. A.: New Morphology-Based Method for Robust Iranian Car Plate Detection and Recognition, International Journal of Computer Theory and Engineering, 2, 1793-8201, 2010.

Kuzmić, T., Ninkov, T., Bulatović, V., Vasić, D., and Davidović, M.: Modern Geodetic Methods with Application in the Environmental Management and its Protection, in: Proceedings of 7th International Symposium on Environmental and Material Flow Management, Bor, Republic of Serbia, 2017.

Lahoti, S., Kefi, M.,Lahoti, A., and Saito, O.: Mapping Methodology of Public Urban Green Spaces Using GIS: An Example of Nagpur City, India, Sustainability, 11, 1-23, 2019.

Lin, T.-Y., Maire, M., Belongie, S., Bourdev, L., Girshick, R., Hays, J., Perona, P., Ramanan, D., Zitnick, C. L., and Dollár, P.: Microsoft COCO: Common Objects in Context, arXiv [preprint], arXiv:1405.0312, 21 February 2015.

Miezianko, R. and Pokrajac, D.: People Detection in Low Resolution Infrared Videos, in: Proceedings of the International Conference IEEE Computer Society Conference on Computer Vision and Pattern Recognition, Alaska, USA, 2008.
Neubeck, A. and Van Gool, L.: Efficient Non-Maximum Suppression, in: Proceedings of the 18th International Conference on Pattern Recognition, Hong Kong, China, 2006.

Radović, M., Aderkwa, O., and Wang, Q.: Object Recognition in Aerial Images Using Convolutional Neural Networks, J. Imaging, 3, 1-9, https://doi.org/10.3390/jimaging3020021, 2017.

Ribaric, S., Ariyaeeinia, A., and Pavesic, N.: Deidentification for privacy protection in multimedia content: A survey, Signal Processing: Image Communication, 47, 131-151, 2016.

Saidallah, M., Taki, F., El Alaoui, A. E. B., and El Fergougui, A.: Classification and comparison of license plates localization algorithms, Signal \& Image Processing: An International Journal, 12, 2, https://doi.org/10.5121/sipij.2021.12201, 2021.

Schneiderman, H. and Kanade, T. A.: Statistical Method for 3D Object Detection Applied to Faces and Cars, in: Proceedings of the IEEE Conf. on Computer Vision and Pattern Recognition, Kauai, USA, 2001.

Sommer, L., Schuchert, T., and Beyerer, J.: Deep Learning Based Multi-Category Object Detection in Aerial Images, in: Proceedings of SPIE "Automatic Target Recognition XXVII", California, 2017.

Sztubecki, J., Bujarkiewicz, A., Derejczyke, K., and Przytuła, M.: Displacement and Deformation Study of Engineering Structures, Open Geosci., 12, 354-362, 2020.

Vasić, D., Davidović, M., Radosavljević, I., and Obradović, Đ.: Panorama images for testing proposed algorithm, FileZilla [data set], available at: https://filezilla-project.org/, last access: 19 November 2021.

Viola, P. and Jones, M. J.: Robust Real-Time Face Detection, Int. J. Comput. Vision, 57, 137-154, 2004.

Vu, V.-T., Bremond, F., Davini, G., and Thonnat, M.: Audio-Video Event Recognition System for Public Transport Security, in: Proceedings of the International conference on Crime Detection and Prevention, London, UK, 2006.

Wang, R., Wu, G., Wang, Q., Yuan, L., Zhang, Z., and Miao, G.: Reversible Data Hiding in Encrypted Images Using Median Edge Detector and Two's Complement, Symmetry, 13, 1-17, https://doi.org/10.3390/sym13060921, 2021.

Wang, S.-Z. and Lee, H.-J.: Detection and Recognition of License Plate Characters with Different Appearances, in: Proceedings of the IEEE International Conference on Intelligent Transportation Systems, Shanghai, China, 2003.

Zhang, L., Lin, L., Liang, X., and He, K.: Is faster R-CNN Doing Well for Pedestrian Detection?, in: Proceedings of 14th ECCV: European Conference on Computer Vision, Amsterdam, the Netherlands, 2016. 